\title{
Ubiquitous Media with UPnP and RFID-Based User Interfaces
}

\author{
Gerrit KALKBRENNER \\ Technische Universität Dortmund, Embedded Systems, Otto Hahn Str. 16, Dortmund, Germany \\ E-mail: gerrit.kalkbrenner@udo.edu \\ Received November 3, 2008; revised March 1, 2009; accepted March 3, 2009
}

\begin{abstract}
The evolution of systems and networks, including PDA, handhelds, mobile phones, WLAN, and Bluetooth provides us new scenarios for media presentation. Because of the growing number of such (personal) devices in the Personal Area Network of the User it is necessary to set up a system, in which the user doesn't lose control over the media and their corresponding presentation devices. Digitalisation will lead us to a split of into content (music, video), storage (e.g. compact discs, server), and user interfaces (receiver). Media will no longer be stored in shelves at home, but in storage spaces located somewhere in the network. New user interfaces like PDA and mobile phones will replace the panel field of an old fashion CD-Player and amplifier. A protocol is required for synchronisation and controlling this media scenario, which is UPnP (Universal Plug and Play).

This paper describes a scenario based on UPnP and its implementation provided by the author.
\end{abstract}

Keywords: Ubiquitous Media, UPnP, RFID

\section{Introduction, Ubiquitous Media Scenario}

In a near future media (Music, Video) will be bought in a spontaneous way, probably by staying in front of a music advertisement table in the Metro or somewhere on the street $[1,2]$. Using our PDA or mobile phone, we just push a button [3]. With location-based service [4] the system knows our place and-based on that information-the system knows the music title on the advertisement table. The music, bought during this procedure, will be transferred via Internet/DSL to our home server or to a central server. This server replaces data stores like CD's or DVD's. Using this server the content will be published in a way that we can access it in a ubiquitous manner. The corresponding cover/booklet will be sent later by letter. The cover is still important, as the human is a haptic oriented being, which likes it to hold something in his hands. Beside this, the cover/booklet contain some important metadata regarding the music/video.

To play the music in a former time we used a CD player, an amplifier and a set of speaker. Those components also serve as a user interface to the media, beside their main electrical function. Therefore, the panel of the CD player builds the user interface for music presentation control. Using different equipment, we get additional user interfaces for the same content. In order to simplify user interfaces other devices like mobile phones and PDA will come into the scenario. [5,6] By pressing a button on my personal PDA, music will be presented on the local speaker, regardless of the given equipment. By moving from the living room to the kitchen, the music will follow with me to the speaker system, which is e.g. installed in the kitchen.

Within this scenario, media content can be distinguished in to 4 components: the 1) media data, (pcmfiles, avi-files) they are stored on a CD, DVD, USB stick, or on a server hard disc. On the other side we have 2) public meta data, which gives information about the title, author and other, the 3 ) personal meta data, which describes the actual storage places, my rights on the media (see DRM) and other, and finally some 4) user interfaces, which allows the control of the presentation of music and video. Beside this, there is the presentation equipment (speaker, projector) and the presentation event itself). 
The central question during the design of a ubiquitous media system is a protocol, which allows the set up, and the dynamic control, e.g. the usage of different user interfaces within a ubiquitous media system.

In this context, the standard UPnP (Universal Plug and Play) [7] got some relevance. This paper gives a practical perspective of a working scenario of ubiquitous media using UPnP.

\section{Problems}

Due to the emerging technology development of wireless networks [8] and hardware for multimedia presentations, applications will change rapidly [9]. Because of the growing number of such (personal) devices in the personal area Network (PAN) of the user, it is necessary to develop a system, in which the user does not lose control over his devices [10]. Digitalisation will lead us to a splitting of content and storage devices (e.g. compact discs, hard disks, flash memory). It will no longer be stored in shelves at home, but in storage spaces located anywhere in the network [11]. Therefore, the storage space available to the user will increase. At the same time, the overview to one's own content will decrease. Upcoming media systems have to grant transparency to the user. The user wants to feel that he knows where his content is located and that he can reach it easily every time, everywhere. Otherwise, users would feel overwhelmed and uncomfortable with those devices. The present situation on the multimedia-market is very much like that.

To avoid these effects a new media system is required, in which the user is the focus of the development. Even though he can reach a much larger amount of content within his system and uses more different devices, he feels comfortable with this system and he has full control over it. This can be achieved by integrating new interaction techniques (as speech-control and gesture recognition in combination with improved graphic user interfaces) in one universal interface.

For unknown reasons, the concepts of Context Awareness [12,13] and Ubiquitous Computing [14,15] are not yet explored for their use in media systems [16]. Both concepts provide us a media system, in which the user is confronted neither with difficult user's manuals nor with changing interfaces.

The actual development of computer indicates some tendencies: devices will decrease in size and the amount of devices available for each user will increase. With wireless networks user will have access to more content. In this paper we develop a concept, within a user can use his media in a comfortable way. Main concepts are abstraction, transparency, and awareness. We can abstract from the physical storage just by simulating them on a known location. First, we will discuss some under laying concepts.

\section{Foundation Concepts, Ubiquitous Media}

The markets for music, video, and games are growing together. Where before we had different markets for each type of media, in the near feature there will be only one, but with much more participants. The market for computer games now days have a larger volume than the market for movies. This is technically enabled by the increasing power of available systems, which today offers more CPU-power and memory and provide multimedia capabilities. In addition, the size of devices is reduced [17].

An implementation of ubiquitous media requires a concept of media transparency. It allows us to access media regardless from its format and its storage place. Media transparency is the main concept for ubiquitous media, so we should take a closer look of it details.

For media transparency, we need:

- Access Transparency-user can access media, regardless from the presentation device, underlying network, and media server.

- Location Transparency-user can access media regardless from its storage place.

- Format Transparency-media content is coded in different formats (mp3, PCM). With format transparency, an abstraction of the format can be achieved. Media player are mainly multi format capable. Non-fitting formats are converted on the fly by other system components.

- DRM Transparency-witch rights a user has on media content is only clearly visible during the buying process. Later the user wants to access media according the rights and he is not willing to engage with DRM aspects.

The user has to be placed into the middle of a ubiquitous media scenario. Around him, we build up a media system, which:

1) can be used in a simple way in every usage situation,

2 ) is working in the background in a unobtrusive way

3) adapts to the necessities of a user

In the next chapters, we will build up a ubiquitous media system.

\section{Requirement Analysis}

Based on the ubiquitous media idea we take a deeper look about the usage scenario and its changes. Based on this we set up a concept of media transparency. Based on this a concept for the dynamic integration of new user interfaces is required. It provides a comfortable access to future media systems; even they provide increased func- 
tionality, more presentation systems and a larger amount of media content. The quality of user interfaces can be increased significantly by considering context awareness. It enables the media system to react on the situation, in which the user currently is involved. In this way, the system can be controlled in an intuitive way.

Critical is the protection of personal information. In order of building a transparent and adaptive system, it needs to collect and store information about the user.

\section{System Design}

The System can be successfully built when separating some aspects, which traditionally are combined tightly. These aspects are 1) user interfaces and system control, 2) media data storage and access, and 3) system management.

By separating and hiding some of these aspects, a system can be build, which partially disappears from the sight of the user. He can concentrate again on his main aim, the consummation of media content.

The system will be build up by the following components:

\subsection{Media Server}

The assignment of the media server will be the storage and delivery of media content. There might be several servers in a system, which holds all content data: mp3-, pcm-, avi-, vob-, and other media files. Special server might be capable to convert media formats into each other. A server might be build up by regular pc hardware, network attached hard drives (NAS), CD/DVD changer, or hard disk based recorder/receiver. Renderer/ presentation systems will access media content via networks (e.g. Ethernet, WLAN, and Bluetooth) and selected protocols (e.g. NFS, SMA, and RTP).

\subsection{Renderer (presentation environment)}

The main task of a rendering system is the access of content from media server and its preparation for presentation. Audio content might be presented via a speaker system, video content via a television set or by a projection system (beamer). Renderer can be build up from regular PC hardware or by special equipment, which fit into the apartment and living ambient. There are special components available, which are build up by a LINUX system and supports the sound and vision platform. These Systems are highly extendable.

\subsection{Control Points}

User interfaces, which are used to control the ubiquitous media system, mainly are built as control points. A control point enables the user to adjust main system parameter like selected media, play, pause, stop, presentation device, and other. Control points can be built up from

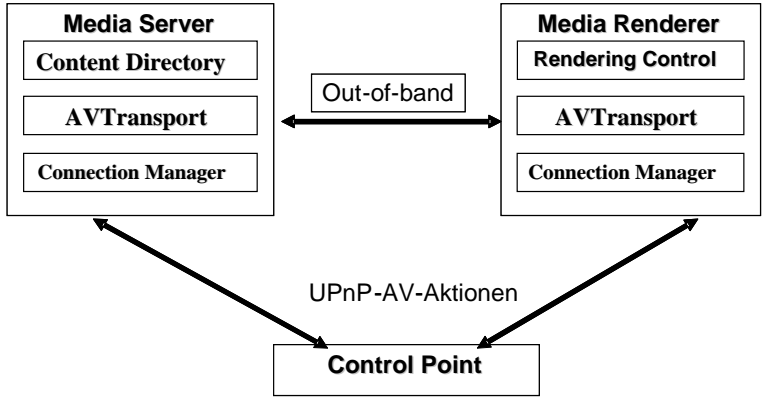

Figure 1. Selecting and controlling media presentation.

regular PC hardware, remote control, PDA, mobile phones or other small devices. However, a control point does not have necessarily a user interface. The phone during a phone call itself might set the music to pause. A control point reacts to user and other events, and in result, it controls some of the system parameter. Figure 1 shows the system components.

\section{System Implementation}

Main components might be implemented in Java, in order of portability. Protocols are implemented using UPnP (Universal Plug and Play) which is based on SOAP, XML and HTTP.

UPnP enables control points to detect server and renderer in a dynamic way. Commands like "music pause"originated on a PDA-are transferred via UPnP to the renderer, which on the other hand notifies the server via UPnP protocol to stop the streaming of content.

\subsection{Detection of System Components}

Control points have to detect server and renderer components, in order to check their facilities and to transmit commands to them.

Based on networks like Bluetooth or WLAN a IP connection will be established. The aim of a DHCP server is to assign IP addresses. As part of the UPnP protocol, a detection request is sent by broadcast. Renderer and server will send back their profiles and tables of offered functions.

UPnP offers an abstraction from IP address to service classes and names, so control points can deal with them on a high-level abstraction.

\subsection{Choosing Media}

The user wants to select media from the offer of his server (single system or a collection). Also bought music (please compare the purchasing scenario from the beginning of this paper) will be placed on this server. A search request is sent to al corresponding server. From the result 
the user can selects music or videos. Figure 2 shows the implementation of a control point on a PDA. Also mobile phones can be used for this purpose.

\subsection{Playing Media}

By selecting a music or video title, the request is sent to the nearest renderer. The renderer requests the media data directly from the server and performs the presentation.

\subsection{Considering the Location of Persons (loca- tion awareness)}

An important aspect in the ubiquitous media scenario is the ubiquitous access of media. If the user moves to a different room, e.g. the music may follow him. Several implementations are conceivable. One solution is a passive infrared sensor system in each room. The problem with this solution consists in a restriction of privacy [12].
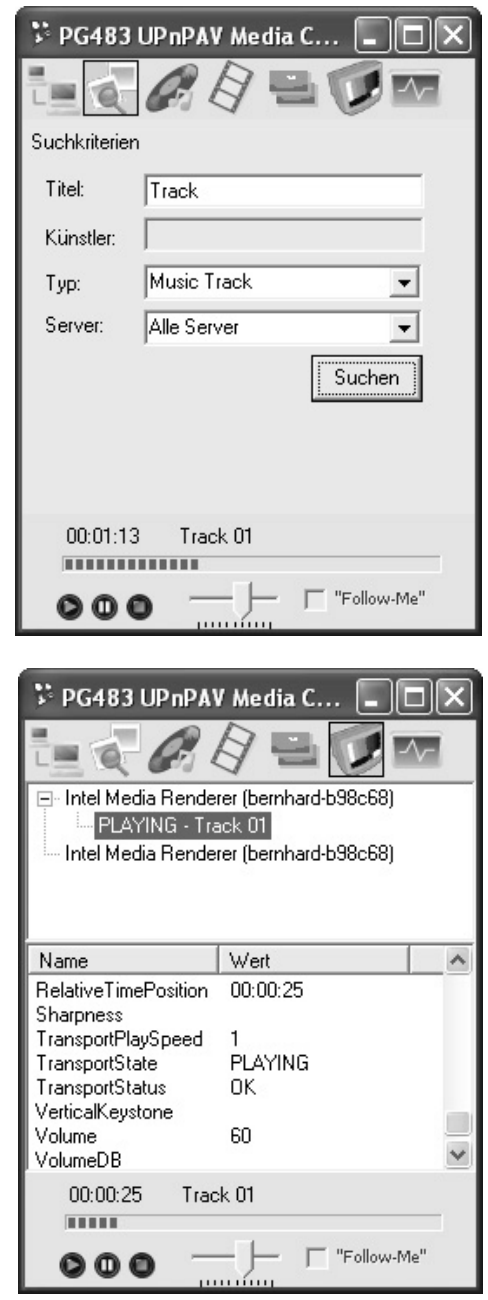

Figure 2. Selecting and controlling media presentation.
Further more the system may fail in case of slow movement, more persons and animals.

We decided to take the position of the currently used Bluetooth access point into consideration, in order to calculate the position of the PDA-and its user. Bluetooth cells are small, so the location precision is sufficient. Using the UPnP architecture an access point can be seen as a server, which provides position data. An automated control point with a minimal user interface tracks this data and-in case of user movement-it instructs the current renderer to stop. Then it directs a secondary renderer e.g. in the kitchen to continue the playback at the interrupted playback position.

\subsection{Scriptable Control Point (Stub)}

The system can also react on other events. In order to keep the implementation simple we developed a scriptable control point. With this, it is easy to implement automated tasks. New Interfaces might be integrated in a simple way.

The system may measure the loudness in the room. A ubiquitous media system should notice this and might adapt the speaker volume.

\subsection{Context awareness (telephone calls)}

A ubiquitous media system should adapt to several events. One type of event are telephone calls. During a phone call, a television transmission should be set to time shift mode. Music and video presentations should be set to pause.

Our implementation uses voice over IP telephony in order to simplify the implementation. We implemented a combination of asterisk VoIP client [18] and the scriptable control point, which stops the music playback. After terminating the call, the playback continues.

\subsection{Magic DVD Cover}

The classical DVD cover has still some importance, as human are orientated in a way that they like to take things with her hand. That's why we like to increase the importance of the DVD cover. We are doing this by adding an RFID tag. By taking a DVD from the shelf and placing it in front of the renderer, this DVD will be discovered by an integrated RFID receiver, which indicates the ubiquitous media system to play the corresponding movie.

\subsection{Cover with Touch Sensitive Elements}




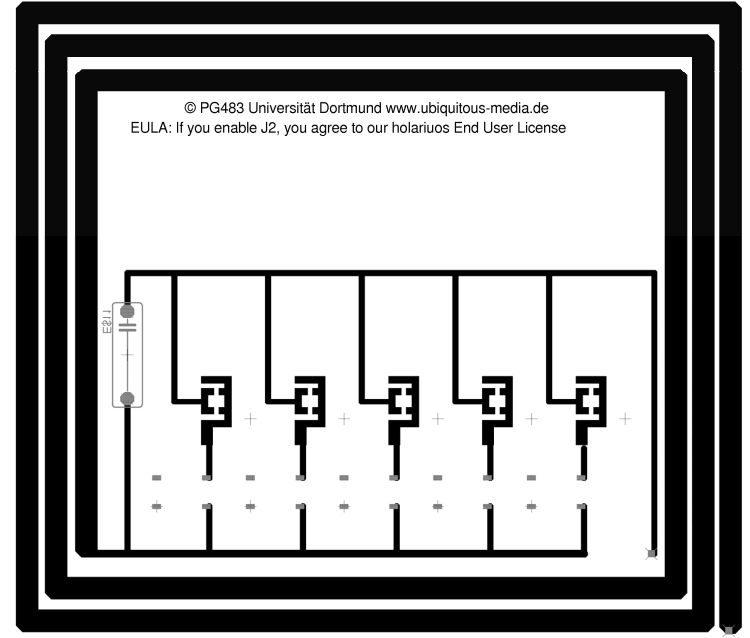

Figure 3. CD-cover integrated switchable RFID-TAG.

In order of introducing new user interfaces, we developed a CD cover with sensitive elements. By touching e.g. the third track on the content list of the CD booklet, the ubiquitous media system will play the according track.

However, it would expect a bit too much if the user has to replace batteries even in his CD booklet. So we have to choose a technique which works without batteries. Therefore, we implemented RFID-Tags in the CD cover. To distinguish between the different music tracks we developed switched RFID tags, which are activated individually by pressing a selected region on the booklet. Figure 3 shows the antenna and fife RFID tags together with the corresponding switches.

A RFID receiver might receive the commands from the RFID tag. This RFID receiver works also as a control point, as it tells the renderer to play a certain music file.

\section{Conclusions}

Within this implementation, we were able to introduce some basic concepts for ubiquitous media. We implemented access transparency, so the user can access media, regardless from the presentation device, underlying network, and media server.

Secondary we implemented location transparency. The user can access media, regardless from its storage place. With this, it is possible to build a "follow me" mode for music. If the user moves to a different room, the music will pause and continue in the other room (location awareness).

And third, we implemented format transparency. The system itself is responsible for content and coding of different formats (mp3, pcm).

We did not implemented DRM transparency, as DRM works against the ubiquitous media concept. The goal of
DRM developer is to fix content to a storage medium or a player, in order of controlling the presentation. Further work is required in order to fulfill DRM transparency by integrating different media player systems.

With our magic DVD cover we came back to haptic based user interfaces. Our CD cover with touch sensitive elements leads us to intuitive user interface. By introducing context awareness a phone call can set the television transmission to time shift.

The protocol UPnP (Universal Plug and Play) gives a good foundation for the implementation of a flexible ubiquitous media system.

\section{References}

[1] J. A. Vince and R. Earnshaw, "Digital media: The Future,” London (Springer), 2000.

[2] F. Schöner, "Multimedia revolution der musik-und medienwirtschaft,” Reinhard Flender, Elmar Lampson, Musik im Internet, Berlin (Kulturverlag Kadmos), pp. S83-S110, 2001.

[3] S. Drews, "Ubiquitous media, vision des digital home der zukunft und anforderungen hinsichtlich seiner realisierung,” Magisterarbeit, Technische Universität Berlin.

[4] N. Dyer and J. Bowskill, "Ubiquitous communications and media-steps toward a wearable learning tool,” J. A. Vince, R. Earnshaw (Eds.), Digital Media: The Future, London (Springer), pp. S61- S74. 2000.

[5] G. Kalkbrenner, "Mobile management of local infrastructure,” Softcom, 2002.

[6] G. Kalkbrenner and F. Nebojsa, "Campus mobil-mobile services for campus and student needs," Softcom, 2002.

[7] http://www.upnp.org/.

[8] Wireless World Research Forum, Book of Visions, Visions of the Wireless World, 2001.

[9] B.-L. Tim, "The World Wide Web: Past, present and future," o.O.

[10] L. Barkhuus and A. Dey , "Is context-aware computing taking control away from the user? Three levels of interactivity examined," Proceedings of UbiComp '03, pp. 150-156, Springer, 2003.

[11] W. Buxton, "Living in augmented reality-ubiquitous media and reactive environments," K. Finn, A. Sellen, S. Wilber, (Eds.), Video, Mediated Communication, Hillsdale N. J. (Erlbaum), pp. 363-384, 1997.

[12] B. N. Schilit, J. Hong, and M. Gruteser, "Wireless location privacy protection computer," Vol. 36, No. 12, pp. 135-137, December 2003.

[13] Special Issue on ContextAware. Computing, Personal and Ubiquitous Computing, Vol. 5, Springer Verlag, 2001.

[14] M. Weiser, "The testbed devices of the infrastructure for ubiqitous computing project,” 1994.

http://ubiq.com/hypertext/weiser/testbeddevices.html. 
[15] Abowd, Gregory: Ubiquitous Computing International Conference, Proceedings, Berlin, Heidelberg, New York, Barcelona 2001.

[16] L. Lessig, "The future of ideas - The fate of the commons in a connected world,” New York (Vintage Books), 2002.
[17] F. Mattern (Ed.), “Total vernetzt Szenarien einer informatisierten Welt,” Berlin, Heidelberg, New York, u. a. (Springer), 2003.

[18] http://www.asterisk.org/. 